\title{
Capturing the Most Active State of a Palladium(0) Cross-Coupling Catalyst
}

Sii Hong Lau, ${ }^{\dagger}$ Liye Chen, ${ }^{\dagger}$ Katherine M. Davis, ${ }^{\ddagger} \|$ lia Kevlishvili, ${ }^{\S}$ Peng Liu, ${ }^{\S}$ and Brad P. Carrow*,\#

${ }^{\dagger}$ Department of Chemistry, Princeton University, Princeton, New Jersey 08544, United States

${ }^{\ddagger}$ Department of Chemistry, Emory University, Atlanta, Georgia 30322, United States

${ }^{\S}$ Department of Chemistry, University of Pittsburgh, Pittsburgh, Pennsylvania 15260, United States

"Department of Chemistry, University of Houston, Houston, Texas 77204, United States

\begin{abstract}
Zerovalent palladium complexes are ubiquitous active species in modern cross-coupling reactions that comprise many premier methods for the construction of $\mathrm{C}-\mathrm{C}$ and $\mathrm{C}$-heteroatom bonds in organic synthesis. While palladium(0) complexes stabilized by two or more dative ligands are widely known, the most active form of $\mathrm{Pd}(0)$ coordinated by a single ancillary ligand ("monoligated $\mathrm{Pd}(0)$ ") has long eluded direct characterization. We report the synthesis and unambiguous solution- and solid-state characterization of functionally 12-electron $\mathrm{Pd}(0)$ complexes coordinated by a single tri(1adamantyl)phosphine ( $\left.\mathrm{PAd}_{3}\right)$ ligand. Access to these fleeting intermediates was achieved by enabling $\mathrm{B}$ to-Pd transmetalation reactions that occur at cryogenic temperature. This work opens new avenues to experimentally interrogate highly reactive on-cycle $\mathrm{Pd}(0)$ catalysts and their structure-dependent reactivity and speciation, which should be broadly informative in continuing studies of catalytic processes featuring the prevalent $\mathrm{Pd}(0) / \mathrm{Pd}(\mathrm{II})$ redox couple.
\end{abstract}

\section{Introduction}

Palladium complexes catalyse an unrivaled diversity of cross-coupling reactions that forge carbon-carbon bonds and carbon-heteroatom (e.g., C-N, C-O, C-S) bonds ${ }^{1}$, which comprise some of the most utilized organometallic reactions in the synthesis of pharmaceutical intermediates, agrochemicals, fine chemicals, and organic electronic materials ${ }^{2}$. The general features of cross-coupling catalytic cycles have been confirmed through extensive experimental and computational mechanistic studies, and factors that affect each elementary step are widely known. A typical reaction mechanism proceeds by transfer of an organic group to the zerovalent catalyst by oxidative addition (OA) of an electrophile, followed by transfer of a second organic group from a nucleophilic reagent by transmetalation (TM) to the catalyst then product formation through bond-forming reductive elimination $(R E)^{3}$. Trapping of a $\operatorname{Pd}(0)$ complex by organic electrophiles or oxidants is a universally conserved first step across all cross-coupling reactions as well as related transformations that rely on a $\mathrm{Pd}(0) / \mathrm{Pd}(\mathrm{II})$ redox couple, such as Tsuji-Trost allylation ${ }^{4}$, carbonylation $^{5}, \mathrm{C}-\mathrm{H}$ functionalization ${ }^{6,7}$, and Wacker-type oxidations ${ }^{8}$. However, the coordinativelyunsaturated active state of zerovalent Pd catalysts bound by a single ancillary ligand lack has long eluded direct experimental characterization in the solution state. Uncertainties about the structure and structuredependent reactivity of such key catalytic intermediates constitute an impediment toward continuing development of more efficient, selective, and sustainable cross-coupling processes. 


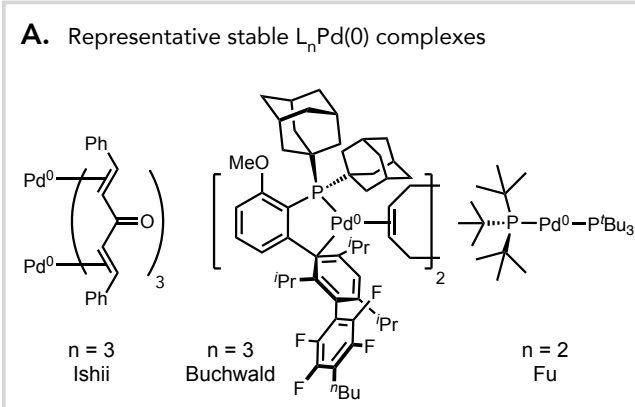

$\geq 2$ Ancillary ligands generally $\cdot$ Off-cycle intermediates
B. Speciation toward active $\mathrm{Pd}(0)$

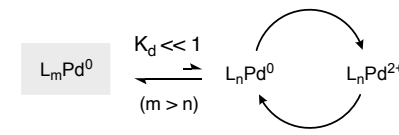

e.g.,

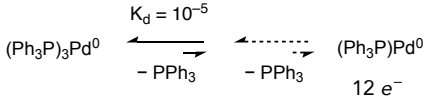

Most active form: "monoligated" ( $n=1)$ Transient $\cdot$ Structually elusive

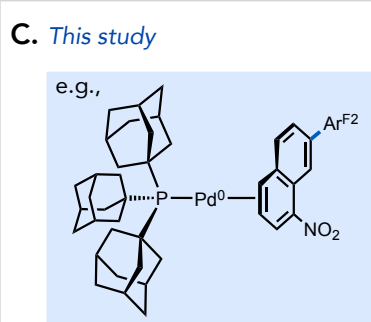

On-Cycle 'Monoligated Pd(0)'

Solution, solid-state characterization

Reactivity profiling

Speciation (e.g., disproportionation)

Figure 1. (A) Illustrative examples of stable $P d(0)$ complexes of the general formula $L_{n} P d(0)$ where $n>1$ and $L=$ dative ligands (e.g., phosphines, alkenes) ${ }^{9-11}$, (B) typical, unfavorable ligand dissociation equilibria to form the most active state of a $\mathrm{Pd}(0)$ catalyst $^{12}$, and $(\mathrm{C})$ structure of an on-cycle $\mathrm{Pd}(0)$ complex coordinated by a single ancillary ligand characterized in this work $\left(\mathrm{Ar}^{2}=2,6-\mathrm{F}_{2} \mathrm{C}_{6} \mathrm{H}_{3}\right)$.

Monoligated $\mathrm{Pd}(0)$ species have been frequently proposed in catalytic cycles and implicated from kinetic studies ${ }^{13}$, but they have been exceedingly difficult to detect due to their coordinatively unsaturated and consequently highly reactive nature ${ }^{14}$. Conversely, $\operatorname{Pd}(0)$ complexes that contain more than one ancillary ligand, such as strong $\sigma$-donors (i.e., phosphines or $\mathrm{N}$-heterocyclic carbenes) or $\pi$-acceptors (alkenes), can be sufficiently stable to isolate (Fig $1 \mathrm{~A})^{9-11,15-18}$, but these complexes are generally inactive in their native form and must undergo one or more thermodynamically unfavorable ligand dissociations to enter a catalytic cycle. These pre-equilibria strongly suppress the concentration of the active $\mathrm{Pd}(0)$ catalyst and throttle catalytic efficiency as a consequence and can even gate selective OA reactions. ${ }^{19}$ Consider the classic cross-coupling catalyst $\left(\mathrm{Ph}_{3} \mathrm{P}\right)_{4} \mathrm{Pd}(0)$ as a representative case, which only reluctantly dissociates phosphine $\left(K_{d} \leq 1.5 \times 10^{-5} \mathrm{M}\right)^{12}$ to generate $\left(\mathrm{Ph}_{3} \mathrm{P}\right)_{2} \mathrm{Pd}(0)$. Assuming additional phosphine dissociation from the bis(phosphine) complex is at least as unfavorable as from the tris(phosphine) complex, the equilibrium concentration of monoligated $\mathrm{Pd}(0)$ generated from $\left(\mathrm{Ph}_{3} \mathrm{P}\right)_{4} \mathrm{Pd}(0)$ would be sub-nanomolar under normal circumstances and thus kinetically inconsequential (Fig 1B). This accounts for the well-documented inability of $\mathrm{PPh}_{3}$-coordinated $\mathrm{Pd}(0)$ to activate electrophiles possessing strong carbon-halogen bonds, such as chloroarenes ${ }^{20,21}$, because $\mathrm{OA}$ of this electrophile class requires access to functionally 12-electron $\operatorname{Pd}(0)^{22}$. On the other hand, modern catalysts competent to promote difficult oxidative addition reactions featured in contemporary cross-coupling methods take advantage of hindered ancillary ligands that disfavor the formation of inactive $\mathrm{Pd}(0)$ species with higher coordination numbers ${ }^{22}$.

It should be of little surprise that monoligated $\mathrm{Pd}(0)$ species have been suggested to be difficult, or even impossible, to characterize unambiguously in solution ${ }^{23}$. The detection of such species in the gas phase by mass spectrometric techniques has been a notable advance in this regard, using either a charge tagged phosphine ligand ${ }^{24}$ as a ligand or by analysis through desorption electrospray ionization mass spectrometry ${ }^{25}$. However, the ionization processes may be sufficiently energetic to eject weakly associated molecules from the metal coordination sphere thus introducing some potential limitation of these methods 
to divine the full solution phase structures. In that regard, theoretical investigations have gleaned insights into the solution phase ${ }^{23,26-29}$. Ab initio molecular dynamic simulations by Lledós suggested $\left(\mathrm{Ph}_{3} \mathrm{P}\right) \operatorname{Pd}(0)$ may exist as a solvent-coordinated species ${ }^{29}$, but to date experimental validation has been lacking. Condensed phase characterization of these species has remained an outstanding challenge and could unlock important new details about their structure and the dependence of stability and reactivity on ancillary ligand identity at this oxidation state. In this study, we report definitive solution spectroscopic and crystallographic characterization of catalytically relevant $\mathrm{Pd}(0)$ complexes coordinated by a single monodentate ancillary ligand $\mathrm{PAd}_{3}(\mathrm{Ad}=1$-adamantyl) (Fig $1 \mathrm{C})$. The use of $\mathrm{PAd}_{3}$, recently reported by our group, was key to accelerating B-to-Pd TM at a sufficiently low temperature to stabilize coordinatively unsaturated $\operatorname{Pd}(0)^{30-34}$.

\section{Results}

A first-generation synthesis of a $\left(\mathrm{Ad}_{3} \mathrm{P}\right) \mathrm{Pd}(0)$ complex was adapted from a previously reported stoichiometric TM reaction by our group, which was shown to occur between the organopalladium cation $\left[\left(\mathrm{PAd}_{3}\right) \mathrm{Pd}\left(\mathrm{p}-\mathrm{FC}_{6} \mathrm{H}_{4}\right)(\mathrm{THF})\right]^{+} \mathrm{BF}_{4}^{-}(1)$ and arylboronic acids even below room temperature (Fig 2$)^{32}$. When excess $\mathrm{C}_{6} \mathrm{~F}_{5} \mathrm{~B}(\mathrm{OH})_{2}$ was added to a THF solution of 1 at $-70{ }^{\circ} \mathrm{C}$, biaryl products were spectroscopically detected as a result of rapid TM then RE. Additionally, analysis by ${ }^{31} \mathrm{P}$ NMR spectroscopy at $-70{ }^{\circ} \mathrm{C}$ indicated complex 1 was cleanly converted to a single new phosphorus-containing product 2 ( $\delta_{P} 95.1$ ppm), which was tentatively assigned to be a $\left(\mathrm{Ad}_{3} \mathrm{P}\right) \mathrm{Pd}(0)$ species based on the concomitant formation of biaryl. While species 2 was persistent at $-70{ }^{\circ} \mathrm{C}$, it gradually disappeared upon raising the solution temperature to $-50{ }^{\circ} \mathrm{C}$. Decomposition to $\left(\mathrm{Ad}_{3} \mathrm{P}\right)_{2} \mathrm{Pd}(0)\left(3, \delta_{\mathrm{P}} 79.1 \mathrm{ppm}\right)$ was detected upon warming to room temperature, which presumably occurs by bimolecular ligand disproportionation between two monoligated species along with formation of a proportional amount of Pd black (Fig 2). In a separate

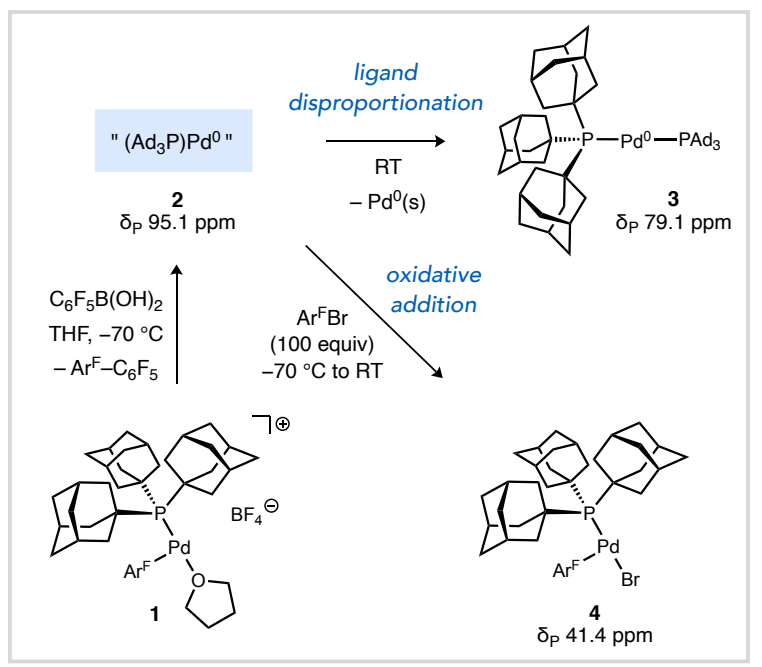

Figure 2. Reactivity profile of 2 generated from cationic TM supports its identity as $\left(\operatorname{Ad}_{3} P\right) \operatorname{Pd}(0)\left(\operatorname{Ar} r^{F}=4\right.$ $\left.\mathrm{FC}_{6} \mathrm{H}_{4}\right)$. 
experiment, a solution of 2 generated with $\mathrm{C}_{6} \mathrm{~F}_{5} \mathrm{~B}(\mathrm{OH})_{2}$ (2 equiv) at $-70{ }^{\circ} \mathrm{C}$ was trapped by addition of the electrophile $p-\mathrm{FC}_{6} \mathrm{H}_{4} \mathrm{Br}$ (100 equiv). Exclusive formation of the known T-shaped oxidative addition complex $\left(\mathrm{Ad}_{3} \mathrm{P}\right) \mathrm{Pd}\left(p-\mathrm{FC}_{6} \mathrm{H}_{4}\right) \mathrm{Br}(4)$ was observed by NMR spectroscopy after warming slowly to room temperature (Fig 2). Both the ligand disproportionation and fast oxidative addition reactions are consistent with the expected high reactivity of an on-cycle $\left(\mathrm{Ad}_{3} \mathrm{P}\right) \mathrm{Pd}(0)$ intermediate.

While preliminary reactions of 2 implicated a potential monoligated $\mathrm{Pd}(0)$ species, several reasonable structures of this complex 2 must be considered, such as mononuclear $\left(\mathrm{Ad}_{3} \mathrm{P}\right) \mathrm{Pd}(0),\left[\left(\mathrm{Ad}_{3} \mathrm{P}\right) \mathrm{Pd}\right]_{\mathrm{n}}$ clusters, $\pi$-arene and solvento complexes (Fig $3 \mathrm{~A}$ ). The large excess of boronic acid required to cleanly form 2 under these conditions complicated initial efforts to fully characterize the monoligated $\mathrm{Pd}(0)$ species. We thus turned to extended X-ray absorption fine structure (EXAFS) to differentiate between plausible structures in solution ${ }^{35,36}$. The collected spectra were interpreted by real-space multiple-scattering theory and compared to the simulated spectra of the proposed structures. The presence of a $\mathrm{Pd}-\mathrm{Pd}$ bond expected for $\mathrm{Pd}(0)$ clusters was ruled out based on the absence of an intense peak at ca. $2.7 \AA$ associated with Pd-Pd scattering in the spectrum of a Pd-foil standard (Fig 3B). A 12-electron mononuclear structure (5) was also excluded because of poor fitting of the simulated spectrum to the experimental data. On the other hand, the simulated spectra for a THF complex 6 and a model $\pi$-arene complex $\left(\mathrm{Ad}_{3} \mathrm{P}\right) \mathrm{Pd}^{0}\left(\eta^{2}-\mathrm{C}_{6} \mathrm{H}_{6}\right)$ both agree well with the experimental data. While these latter two structures are not readily differentiated by the EXAFS measurements, other experimental and computational studies were conducted to illuminate important structural details of the zerovalent species.

Density functional theory (DFT) calculations were performed to interrogate the relative ground-state energies of a variety of potential solvento and $\pi$-arene complexes. Optimization of structures with general formula $\left(\mathrm{Ad}_{3} \mathrm{P}\right) \mathrm{Pd}^{0}(\mathrm{THF})_{n}(\mathrm{n}=1-3)$ led to convergence of higher coordinate 16 - and 18-electron species to the low coordinate complex $\left(\mathrm{Ad}_{3} \mathrm{P}\right) \mathrm{Pd}^{0}(\mathrm{THF})(6)$, which is $9.6 \mathrm{kcal} / \mathrm{mol}$ lower in free energy than mononuclear $\left(\mathrm{Ad}_{3} \mathrm{P}\right) \mathrm{Pd}(0)$ species 5 (Fig $\left.3 \mathrm{~A}\right)$. Similarly, optimization of putative 18-electron $\left(\mathrm{Ad}_{3} \mathrm{P}\right) \mathrm{Pd}(0)\left(\eta^{6}-\right.$ arene) complexes (arene $=2,3,4,4^{\prime}, 5,6$-hexafluorobiphenyl (HFB) or pentafluorophenylboronic acid) converged to low coordinate $\eta^{2}$-arene species 7 or 8 that are 11.9 and $11.3 \mathrm{kcal} / \mathrm{mol}$ lower in free energy than 5, respectively (see ESI for structures and energies of other solvento or $\pi$-arene $\mathrm{Pd}(0)$ complexes). Consistent with EXAFS measurements, the DFT data support 14-electron $\operatorname{Pd}(0)$ complexes as thermodynamically most favorable. The calculated free energies of the $\left(\mathrm{Ad}_{3} \mathrm{P}\right) \mathrm{Pd}(\mathrm{THF})$ 6, $\left(\mathrm{Ad}{ }_{3} \mathrm{P}\right) \mathrm{Pd}\left(\eta^{2} \mathrm{C} 2{ }^{\prime}, \mathrm{C} 3^{\prime}-\mathrm{HFB}\right) \quad 7$, and $\left(\mathrm{Ad}{ }_{3} \mathrm{P}\right) \mathrm{Pd}\left(\eta^{2} \mathrm{C} 1, \mathrm{C}-\mathrm{C}_{6} \mathrm{~F}_{5} \mathrm{~B}(\mathrm{OH})_{2}\right) 8$ differ by only ca. $2 \mathrm{kcal} / \mathrm{mol}$ thus precluding clear differentiation between these candidate structures. Related $\left(\mathrm{Ad}_{3} \mathrm{P}\right) \mathrm{Pd}(0)$ complexes coordinated by an aryl halide (e.g., 9) also favor the $\eta^{2}$-coordination mode (Fig 3C), similar to 7 and 8. Interconversion of 9 to an $\eta^{1}$-arene species (10) by $\pi$-walking is facile and nearly isoergic, which facilitates concerted oxidative addition with an energy barrier of only $\Delta G^{\ddagger}{ }_{203}=7.5 \mathrm{kcal} / \mathrm{mol}$ relative to the $\eta^{2}$-arene ground state. These data suggest arene coordination to $\left(\mathrm{Ad}_{3} \mathrm{P}\right) \mathrm{Pd}(0)$ is dynamic and the monoligated $\pi-$ arene complexes are highly reactive toward $O A$, as expected. 

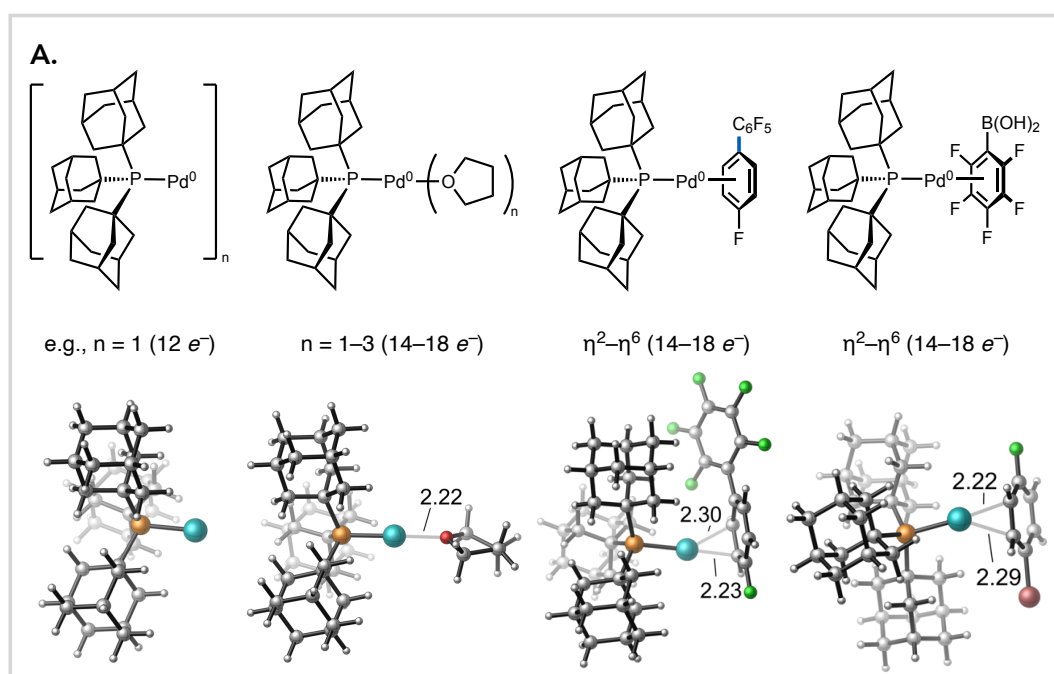

$\mathrm{n}=1-3\left(14-18 e^{-}\right)$

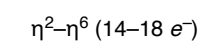

$\eta^{2}-\eta^{6}\left(14-18 e^{-}\right)$
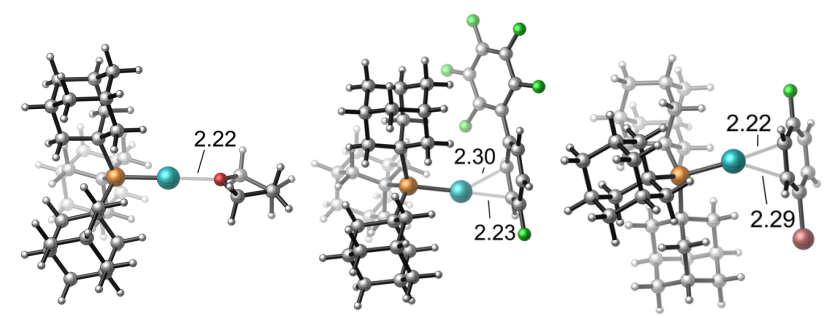

$5(n=1)$

$0.0(0.0)$

\begin{abstract}
$6(n=1)$ $-9.6(-16.9)$
\end{abstract}

$7\left(\eta^{2} C 2^{\prime}, C 3\right)$ $-11.9(-22.4)$
B.

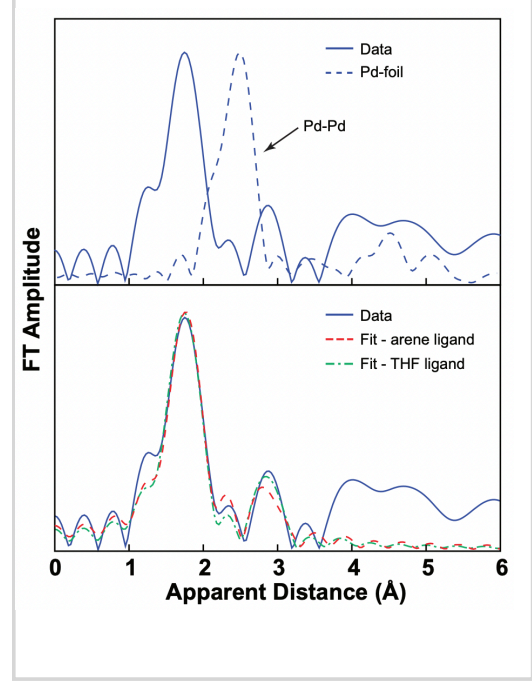

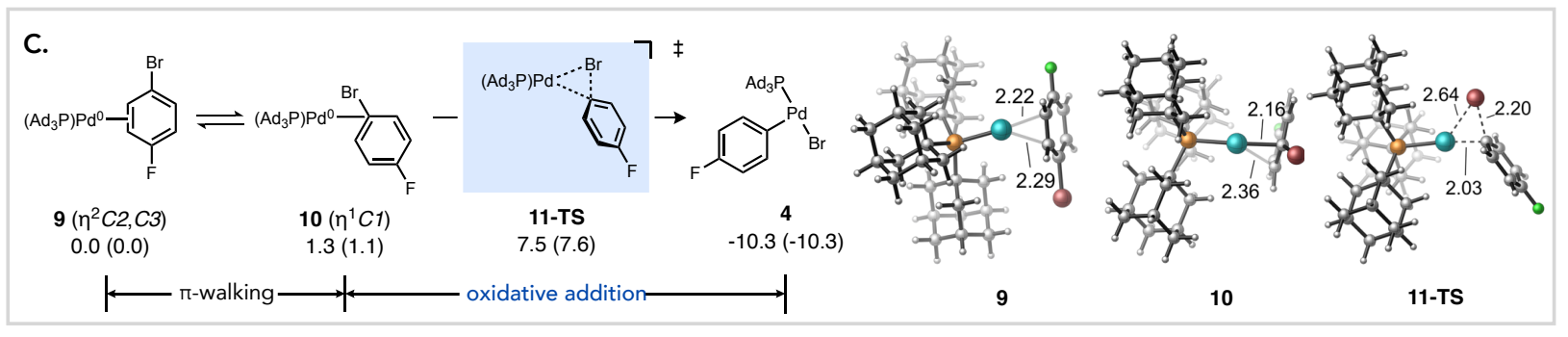

Figure 3. (A) Plausible structures for the $P d(0)$ product formed in Fig 2 and lowest free energy DFToptimized structures. (B) EXAFS spectra of the $\mathrm{Pd}(0)$ species (solid blue line) and best-fit interatomic distances for Pd foil (dashed blue line), $\left(\mathrm{Ad}_{3} \mathrm{P}\right) \mathrm{Pd}^{0}(\mathrm{THF})$ (dashed green line), or $\left(\mathrm{Ad}_{3} \mathrm{P}\right) \mathrm{Pd}^{0}\left(\eta^{2}-\mathrm{C}_{6} \mathrm{H}_{6}\right)$ (dashed red line) species. (C) Calculated energetics for oxidative addition to $\left(\mathrm{Ad}_{3} \mathrm{P}\right) \mathrm{Pd}(0)$. Free energies (enthalpies) in $\mathrm{kcal} / \mathrm{mol}(203.2 \mathrm{~K})$; distances in Å. M06/6-311+G(d,p)-SDD/SMD(THF)//B3LYP-D3/6-31G(d)-SDD.

An alternative synthetic pathway not dependent on large excess of boron reagent, which complicated NMR spectroscopic analysis, was desirable to facilitate structural disambiguation of the low coordinate $\mathrm{Pd}(0)$ species. We took inspiration from studies of protodeboronation (PDB) of arylboronic acids, which by the isolobal analogy is mechanistically parallel to B-to-Pd TM by transposition of a proton for Pd. The relative rate of PDB measured by Lloyd-Jones and coworkers is dramatically higher $\left(k_{\mathrm{rel}}=10^{8}\right)$ upon substitution of a monofluoro (para-substituted) for a pentafluoro arylboronic acid (Fig 4A) $32,37,38$. With this fluorine effect in mind, Denmark and coworkers previously established stoichiometric TM of $\left[\mathrm{Pd}^{2}\left(\mathrm{P}^{t} \mathrm{Bu}_{3}\right)(\mathrm{p}\right.$ $\left.\left.\mathrm{C}_{6} \mathrm{H}_{4} \mathrm{~F}\right)(\mu-\mathrm{OH})\right]_{2}$ (12) with $p-\mathrm{FC}_{6} \mathrm{H}_{4} \mathrm{~B}(\mathrm{OH})_{2}$ arrested at a pre-transmetalation Pd-boronate complex at $\leq-$ $60{ }^{\circ} \mathrm{C}$ (Fig 4B), which facilitated the first characterization of those catalytic interemediates ${ }^{39,40}$. We found that a pre-transmetalation intermediate was not detected upon treatment of complex 12 with $\mathrm{C}_{6} \mathrm{~F}_{5} \mathrm{~B}(\mathrm{OH})_{2}$ rather than $p-\mathrm{FC}_{6} \mathrm{H}_{4} \mathrm{~B}(\mathrm{OH})_{2}$ in toluene/THF at $-70{ }^{\circ} \mathrm{C}$. Instead, we observed complete consumption of 12 , biaryl formation in excellent yield (93\%), and the formation of a new phosphorus-containing product 13 (49\% yield, $\delta_{p}=93.5 \mathrm{ppm}$ ) along with several unidentified side products (Fig 4B). Complex 13 was 
assigned to be a $\left({ }^{\mathrm{B}} \mathrm{Bu} \mathrm{u}_{3} \mathrm{P}\right) \mathrm{Pd}(\mathrm{O})$ species analogous to 2 based on the similarity of ${ }^{31} \mathrm{P} \mathrm{NMR}$ chemical shifts (and the general similarity between $\delta_{P}$ values of other known $\mathrm{Pd}$ congeners coordinated by $\mathrm{P}^{t} \mathrm{Bu}_{3}$ or $\mathrm{PAd}_{3}$ ).
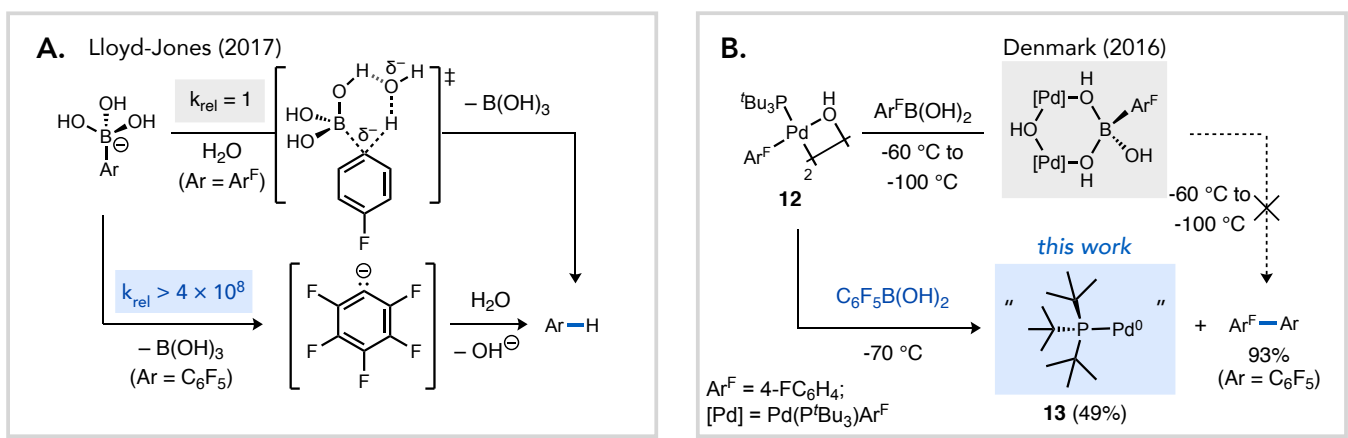

Figure 4. (A) Large substituent effect on rates of protodemetalation (B-to- $\mathrm{H}^{+}$transfer) that, by the isolobal analogy, inspired a strategy for (B) accelerated B-to-Pd TM via the classic hydroxo pathway to access detectable levels of monoligated $\mathrm{Pd}(0)$ species in solution.

Improved chemical stability of coordinated $\mathrm{PAd}_{3}$ over $\mathrm{P}^{\mathrm{t} B \mathrm{Bu}_{3}}$ toward certain metal-mediated decomposition pathways has been noted previously ${ }^{30}$. We thus synthesized an analogue of $\mathbf{1 2}$, $\left[\mathrm{Pd}\left(\mathrm{PAd}_{3}\right)\left(\mathrm{p}_{-}-\mathrm{CF}_{3} \mathrm{OC}_{6} \mathrm{H}_{4}\right)(\mu-\mathrm{OH})\right]_{2} \quad(14)$, to test whether formation of side products is suppressed by this ligand exchange to potentially aid attempted isolation of a low coordinate $\mathrm{Pd}(0)$ species. We observed that reaction of complex 14 with $\mathrm{C}_{6} \mathrm{~F}_{5} \mathrm{~B}(\mathrm{OH})_{2}$ indeed afforded a single observable species by ${ }^{31} \mathrm{P}$ NMR $\left(\delta_{\mathrm{P}}\right.$ $=91.6 \mathrm{ppm}, 75 \%$, Fig 5A) under otherwise identical conditions that generated a mixture of products from

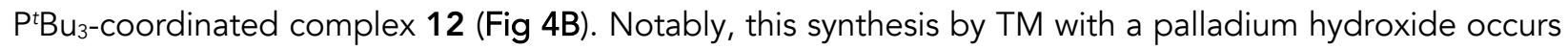
cleanly to form a product (15) even with reduced boronic acid loading compared to those in the earlier cationic TM reactions at low temperature. Peak broadening is observed in the ${ }^{31} \mathrm{P}$ NMR spectrum when the reaction is conducted in THF but without added toluene, which leads us to propose the product 15 is a toluene adduct of $\left(\mathrm{Add}_{3} \mathrm{P}\right) \mathrm{Pd}(0)$. Addition of $p-\mathrm{FC}_{6} \mathrm{H}_{4} \mathrm{Br}\left(120\right.$ equiv) to a solution of $\left(\mathrm{Ad}_{3} \mathrm{P}\right) \mathrm{Pd}(0)$ in $\mathrm{THF}$ at $78{ }^{\circ} \mathrm{C}$ led to formation of the oxidative addition complex 4 in excellent yield (96\%) within 1 min upon warming to $-50^{\circ} \mathrm{C}$. The facile reaction from the $p$-fluorobromobenzene-coordinated species is qualitatively consistent with the low calculated oxidative addition barrier (vide supra). Despite repetitive attempts, we were unable to characterize the labile solvent ligand or to obtain single crystals of $\mathbf{1 5 .}$

The behavior of $\left(\mathrm{Ad}_{3} \mathrm{P}\right) \mathrm{Pd}(0)$, generated from 14, was subsequently tested in the presence of additives (20 equiv each) such as phenanthrene, 1,3,5-tris(trifluoromethyl)benzene, or 1-nitronaphthalene to aid crystallization and structure characterization. Upfield ${ }^{31} \mathrm{P}$ NMR chemical shifts (81-84 ppm) were observed suggesting favorable coordination of these exogenous arenes to the Pd complexes (see ESI, Fig S61-S64). The phenanthrene-containing Pd species (16) in particular was persistent under nitrogen at $-80{ }^{\circ} \mathrm{C}$ over 3 days, which was a sufficient period of time to obtain a single crystal suitable for analysis by $\mathrm{X}$-ray diffraction. The solid-state structure of 16 confirmed this complex to be $\left(\mathrm{Ad}_{3} \mathrm{P}\right) \mathrm{Pd}^{0}\left(\eta^{2} \mathrm{C} 33, \mathrm{C} 46\right.$-phenanthrene) shown in Fig 5A consistent with the favorability of an $\eta^{2}$-arene structure from earlier EXAFS and DFT data. The P- 
$\mathrm{Pd}$-centroid angle of $169.5^{\circ}$ indicates complex 16 adopts a slightly distorted linear, two-coordinate geometry.

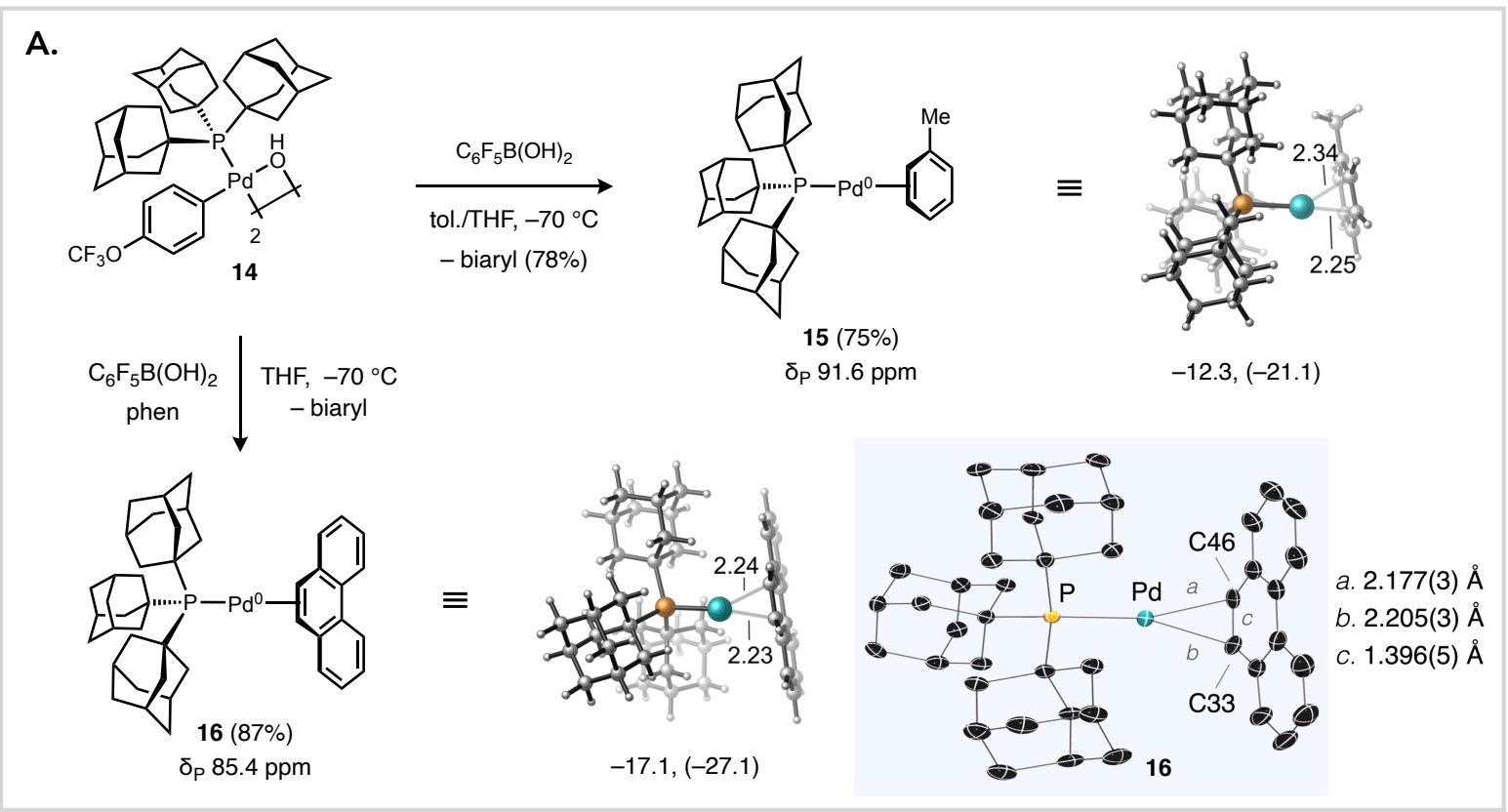

B.
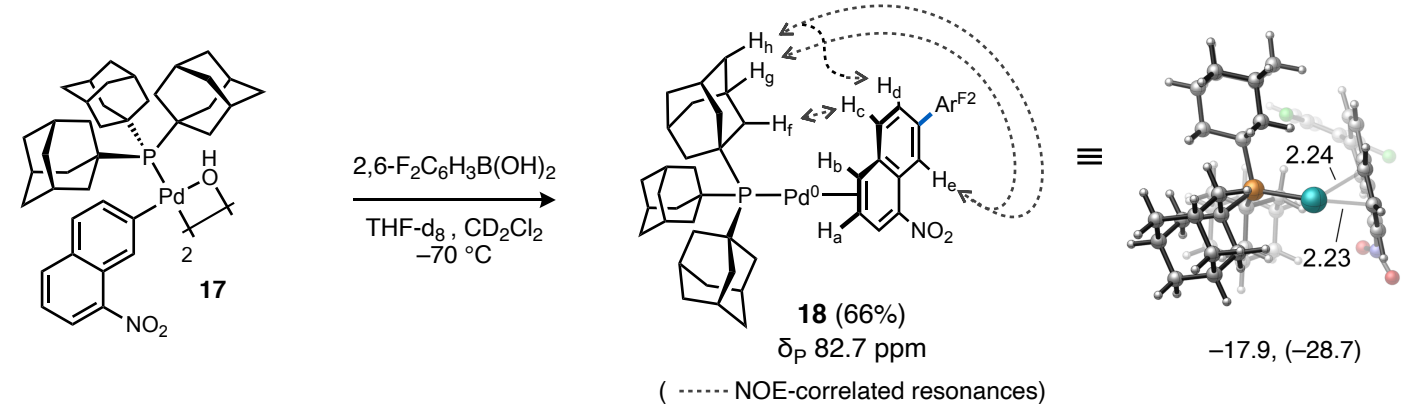

Figure 5. (A) Second-generation route to $\left(\mathrm{Ad}_{3} \mathrm{P}\right) \mathrm{Pd}(\mathrm{O})$ species by the hydroxo pathway for $\mathrm{TM}$; synthesis of complex 16 in the presence of phenanthrene and its solid-state structure (thermal ellipsoids shown at 50\% probability; $\mathrm{H}$ atoms omitted), (B) Synthesis and NMR spectroscopic characterization of an on-cycle, product-bound catalyst 18. Free energies (enthalpies) in $\mathrm{kcal} / \mathrm{mol}(203.2 \mathrm{~K})$ and normalized to 5; distances in $\AA$. M06/6-311+G(d,p)-SDD/SMD(THF)//B3LYP-D3/6-31G(d)-SDD $\left(\mathrm{Ar}^{2}=2,6-\mathrm{F}_{2} \mathrm{C}_{6} \mathrm{H}_{3}\right)$.

Analysis of geometric parameters for this complex also provided insight into the nature of the bonding between Pd and the coordinated arene, which appears to be weaker than coordination of alkenes in stable $\mathrm{Pd}(0)$ complexes. The bond length of C33-C46 in complex $16(1.396(5) \AA)$ is slightly longer than that in free phenanthrene $(1.372 \AA)^{41}$ but shorter than that in $\left(\mathrm{Et}_{3} \mathrm{P}\right)_{2} \mathrm{Ni}^{0}\left(\eta^{2}\right.$-phenanthrene) $(1.428(2) \AA)^{42}$, which is consistent with relatively weaker $\pi$ back-bonding from $\mathrm{Pd}(0)$ to arene. Furthermore, the $\mathrm{Pd}-\mathrm{C} 33$ and $\mathrm{Pd}-$ C46 bond lengths in 16 (2.205(3) and 2.177(3) $\AA$, respectively) are close to the values (2.23 $\AA$ and $2.24 \AA$ ) calculated using DFT. These values are longer than metal-carbon bonds in common palladium $(0) \pi$-alkene 
complexes (ca. 2.04-2.13 $\AA)^{43}$. This likely reflects weaker bonding of an arene to $\mathrm{Pd}(0)$ versus common $\pi-$ acceptors, such as dibenzylideneacetone (dba) and 1,5-cyclooctadiene (see examples in Fig 1A).

Calculation of Ittel-Ibers parameters ${ }^{44}$ provided an additional quantitative assessment of back-bonding based on the nonplanarity of coordinated phenanthrene carbons in the solid-state structure of 16 (see ESI for full details). The value $\alpha$ is defined as the angle between the normals to the planes comprised of the substituent groups bonded to C33 or C46 in coordinated phenanthrene; $\beta$ and $\beta^{\prime}$ are defined as the angles between the C33-C46 bond and C33 or C46 substituent planes, respectively. Increasing values of $a$ and decreasing values of $\beta$ and $\beta^{\prime}$ reflect enhanced metal back-bonding that bends the ligand substituents away from the metal center. The a value is $40.5^{\circ}$ for complex 16 , which is lower than the aforementioned $\left(\mathrm{Et}_{3} \mathrm{P}\right)_{2} \mathrm{Ni}^{0}\left(\eta^{2}\right.$-phenanthrene) complex $\left(44.2^{\circ}\right)$ and significantly less than for a representative $\pi$-alkene complex (1,5-cyclooctadiene) ${ }_{2} \operatorname{Pd}(0)\left(50.2^{\circ}\right)$. Likewise, the angles $\beta$ and $\beta^{\prime}$ are $70.4^{\circ}$ and $69.1^{\circ}$ for 16 and are comparably larger than the respective angles in the aforementioned $\mathrm{Ni}(0)$ complex $\left(67.6^{\circ}\right.$ and $\left.68.3^{\circ}\right)$ or in $(\mathrm{COD})_{2} \mathrm{Pd}(0)\left(64.7^{\circ}\right.$ and $\left.65.2^{\circ}\right)$. The smaller $\alpha$ and larger $\beta$ and $\beta^{\prime}$ values for 16 reinforce that $\pi$ backbonding to coordinated phenanthrene is relatively weak in this monoligated $\operatorname{Pd}(0)$ complex.

We also observed solution-state behavior supporting weak, dynamic coordination of arene. The NMR resonances corresponding to protons attached to C33 and C46 of phenanthrene within 16 were shifted significantly upfield $\left(\Delta \delta_{H}>1 \mathrm{ppm}\right)$ relative to free phenanthrene. This facilitated analysis by Exchange Spectroscopy (EXSY) NMR, which indicated bound and free phenanthrene undergo fast exchange on the NMR time scale at $-70^{\circ} \mathrm{C}$. Together these spectroscopic data along with geometric (Ittel-lbers) parameters support the notion that $\pi$-arene association to $\left(\mathrm{Ad}_{3} \mathrm{P}\right) \mathrm{Pd}(0)$ is dynamic and can be considered distinct from the tightly binding $\pi$-alkenes of most $\mathrm{Pd}(\mathrm{O})$ and $\mathrm{Ni}(\mathrm{O})$ complexes. This also may help to rationalize the highly reactive nature of $\left(\mathrm{Ad}_{3} \mathrm{P}\right) \mathrm{Pd}^{0}$ species compared to more stable $\mathrm{Pd}(0)$ complexes, such as the illustrative examples shown in Fig $1 \mathrm{~A}$.

The structure of a biaryl-bound $\mathrm{Pd}(0)$ species that is formed immediately after RE - a true on-cycle (active) form of a contemporary Suzuki-Miyaura coupling (SMC) catalyst - was also unambiguously characterized in solution. Because coordination of 1-nitronaphthalene to $\left(\mathrm{Ad}_{3} \mathrm{P}\right) \mathrm{Pd}(0)$ was more favorable than solvent coordination (vide supra), we postulated a biaryl containing a 1-nitronaphthalene fragment ${ }^{45}$ that is generated from $\mathrm{C}-\mathrm{C}$ bond forming RE might associate to $\left(\mathrm{Ad}_{3} \mathrm{P}\right) \mathrm{Pd}(0)$ long enough to characterize in solution. A suitable palladium hydroxo complex $\left[\mathrm{Pd}\left(\mathrm{PAd}_{3}\right)(8-\text { nitronaphthalyl) }(\mu-\mathrm{OH})]_{2}(17)\right.$ was prepared to test this hypothesis. Reaction between 17 and 2,6- $\mathrm{F}_{2} \mathrm{C}_{6} \mathrm{H}_{3} \mathrm{~B}(\mathrm{OH})_{2}$ (5 equiv) in THF- $d_{8}$ and $\mathrm{CD}_{2} \mathrm{Cl}_{2}$ at $70{ }^{\circ} \mathrm{C}$ occurred to form a new product 18 as observed by ${ }^{31} \mathrm{P} N M R\left(\delta_{\mathrm{P}}=82.7 \mathrm{ppm}\right.$ ) in $66 \%$ yield (Fig 5B).

The structure of 18 was determined by ${ }^{1} \mathrm{H}$ NMR spectroscopy, ${ }^{1} \mathrm{H}-{ }^{1} \mathrm{H}$ correlated spectroscopy (COSY) and nuclear Overhauser effect spectroscopy (NOESY). Nuclear Overhauser effect between protons $H_{c}, H_{d}$ and $\mathrm{H}_{\mathrm{e}}$ (shown in Fig 5B) in the biaryl ligand, 7-(2,6-difluorophenyl)-1-nitronaphthalene (FPNN), and protons $\mathrm{H}_{\mathrm{f}}, \mathrm{H}_{\mathrm{g}}$, and $\mathrm{H}_{\mathrm{h}}$ in $\mathrm{PAd}_{3}$ confirms 18 to be a biaryl-bound $\left(\mathrm{Ad}_{3} \mathrm{P}\right) \mathrm{Pd}(0)$ complex. The resonances for protons $H_{a}$ and $H_{b}$ are shifted upfield by $\Delta \delta_{H} 2.08$ and 1.54 ppm, respectively, relative to free FPNN; the 
chemical shifts of other protons on the naphthyl fragment in 18 are not significantly changed upon coordination $\left(\Delta \delta_{H}<0.23 \mathrm{ppm}\right)$. These data are consistent with $\mathrm{Pd}$ coordination to $\mathrm{C} 3$ and $\mathrm{C} 4$ in the naphthyl ring distal to the nitro group and corroborate the NOESY structural assignment of 18 as a $\pi-$ arene complex $\left(\mathrm{Ad}_{3} \mathrm{P}\right) \mathrm{Pd}^{0}\left(\eta^{2} \mathrm{C} 3, \mathrm{C} 4-\mathrm{FPNN}\right)$ with $\mathrm{Pd}$ bound to the less hindered side of the FPNN naphthyl ring. As observed for complex 16, we also observed fast exchange between Pd-bound and free FPNN on the NMR time scale at $-70^{\circ} \mathrm{C}$ as determined by EXSY NMR. These data are indicative of labile coordination of FPNN to $\left(\mathrm{Ad}_{3} \mathrm{P}\right) \mathrm{Pd}(0)$ complexes and suggest complex 18 and related $\left(\mathrm{Ad}_{3} \mathrm{P}\right) \mathrm{Pd}^{0}\left(\eta^{2}\right.$-arene) complexes can be regarded as functionally monoligated 12-electron species at ambient temperatures, such as under typical conditions for catalytic cross-coupling reactions.

Lastly, we confirmed that the biaryl FPNN was cleanly formed in excellent yield (88\%) when the SMC between 1-bromo-9-nitronaphthalene and 2,6- $\mathrm{F}_{2} \mathrm{C}_{6} \mathrm{H}_{3} \mathrm{~B}(\mathrm{OH})_{2}$ was conducted using complex 4 as a catalyst under our previously reported conditions ${ }^{33}$. This supports the conclusion that complex 18 represents the product-bound form of an on-cycle $\mathrm{Pd}(0)$ catalyst formed immediately after turnover of a catalytic cycle. To our knowledge, this is the first unambiguous structural characterization of a post-reductive elimination $\operatorname{Pd}(0)$ intermediate coordinated by a single ancillary ligand. Access to such monoligated $\operatorname{Pd}(0)$ intermediates in solution could thus provide new opportunities to study catalytic phenomena not previously possible in an experimental context and elucidate new mechanistic information relevant to contemporary challenges in cross-coupling, such as fidelity control of catalyst transfer in cross-coupling polycondensation ${ }^{46-48}$ and ligand-dependent site selectivity in $\mathrm{OA}$ as a few representative examples ${ }^{11,49,50}$.

\section{Conclusion}

We report the first solution spectroscopic and crystallographic characterization of monoligated $\operatorname{Pd}(0)$ complexes, which unambiguously addresses outstanding questions about the existence and structure of very low coordinate $\mathrm{Pd}(0)$ intermediates involved in contemporary palladium-catalysed cross-coupling reactions ${ }^{24}$. The synthesis and characterization of on-cycle (active) $\left(\mathrm{Ad}_{3} \mathrm{P}\right) \mathrm{Pd}(0)$ intermediates was accomplished by leveraging exceptionally facile transmetalation (TM) reactions at cryogenic temperatures beginning from T-shaped organopalladium complexes. The structure of these catalytic intermediates in solution was determined from EXAFS, DFT computational, and multi-nuclear one- and two-dimensional NMR spectroscopic analyses in addition to single crystal X-ray diffraction. Collectively these data suggest $\left(\mathrm{Ad}_{3} \mathrm{P}\right) \mathrm{Pd}(\mathrm{O})$ complexes adopt exclusively low coordination states and associate only weakly with arene or solvent molecules in solution. These $\left(\mathrm{Ad}_{3} \mathrm{P}\right) \mathrm{Pd}(0)$ species may thus be considered as functionally $12-$ electron species, which helps to rationalize their exceptional reactivity in catalytic processes. We anticipate these results will be broadly informative in continuing investigations of modern cross-coupling reactions. 


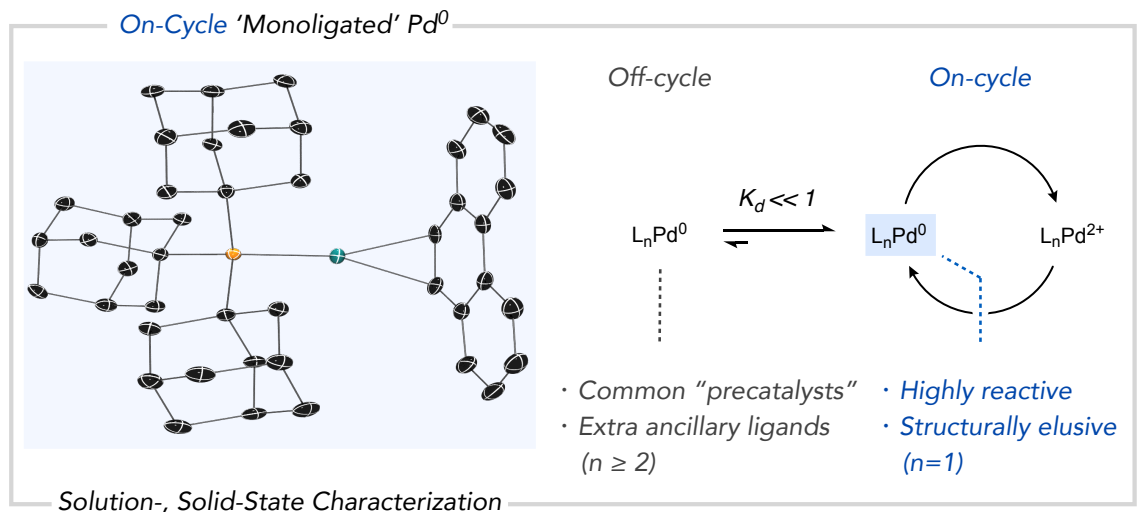

\section{Methods}

Representative synthesis of $\left(\mathrm{Ad}_{3} \mathrm{P}\right) \mathrm{Pd}(0)$ by cationic transmetalation. A solution of complex 1 was first generated as follows: $\mathrm{Pd}\left(\mathrm{PAd}_{3}\right)\left(\mathrm{p}-\mathrm{FC}_{6} \mathrm{H}_{4}\right) \mathrm{Br}, 4(9.0 \mathrm{mg}, 12.5 \mu \mathrm{mol})$ was dissolved in THF $(2.5 \mathrm{~mL})$ in a $4 \mathrm{~mL}$ vial inside an inert atmosphere glove box. In a separate $4 \mathrm{~mL}$ vial, $\mathrm{AgBF}_{4}(19.5 \mathrm{mg}, 100 \mu \mathrm{mol})$ was dissolved in THF (2 mL). Both solutions were chilled at $-35{ }^{\circ} \mathrm{C}\left(\mathrm{MeOH} / \mathrm{H}_{2} \mathrm{O}\right.$ dry ice bath). An aliquot of the latter $\mathrm{AgBF}_{4}$ solution $(50 \mu \mathrm{L}, 2.5 \mu \mathrm{mol})$ was added to the solution of $4(2.5 \mu \mathrm{mol}, 0.5 \mathrm{~mL})$ in a $4 \mathrm{~mL}$ vial at $-35^{\circ} \mathrm{C}$. The mixture was quickly shaken and then left in the cooling bath for $10 \mathrm{~min}$ to ensure full conversion to the cationic complex 1. Separately, an aliquot of a $100 \mathrm{mM}$ solution of $\mathrm{C}_{6} \mathrm{~F}_{5} \mathrm{~B}(\mathrm{OH})_{2}$ in THF $(40 \mu \mathrm{mol}, 0.40$ $\mathrm{mL}, 40$ equiv) was transferred to an NMR tube under nitrogen and cooled at $-78^{\circ} \mathrm{C}$ (acetone/dry ice bath). An aliquot of the solution of $1(1 \mu \mathrm{mol}, 0.20 \mathrm{~mL})$ was then added via syringe to the NMR tube maintained at $-78{ }^{\circ} \mathrm{C}$. The mixture was shaken vigorously for $10 \mathrm{~s}$, let stand for $10 \mathrm{~min}$ at $-78{ }^{\circ} \mathrm{C}$, then inserted into the NMR probe. Complete conversion of 1 was determined from ${ }^{19} \mathrm{~F}$ and ${ }^{31} \mathrm{P}\left\{{ }^{1} \mathrm{H}\right\} N M R$ spectra at $-70{ }^{\circ} \mathrm{C}$; complex 2 was generated as the only observable phosphorus-containing product as determined by ${ }^{31} \mathrm{P}\left\{{ }^{1} \mathrm{H}\right\}$ NMR spectroscopy.

Representative synthesis of $\left(\mathrm{Ad}_{3} \mathrm{P}\right) \mathrm{Pd}(0)$ by neutral "hydroxo" transmetalation. [Pd( $\left.\mathrm{PAd}_{3}\right)\left(\mathrm{p}-\mathrm{CF}_{3} \mathrm{OC}_{6} \mathrm{H}_{4}\right)(\mu-$ $\mathrm{OH})]_{2}, 14(5.4 \mathrm{mg}, 3.75 \mu \mathrm{mol})$ and arene $\left(7.5 \mu \mathrm{mol}, 1\right.$ equiv to $\left.[\mathrm{Pd}]_{\text {total }}\right)$ were dissolved in THF- $\mathrm{d}_{8}(0.4 \mathrm{~mL})$ in a $4 \mathrm{~mL}$ vial inside an inert atmosphere glove box. The resulting solution was transferred into an NMR tube containing a sealed capillary tube containing external standard $\left(\mathrm{PCl}_{3}\right.$ and $\mathrm{C}_{6} \mathrm{~F}_{7}$ in $\left.\mathrm{CD}_{2} \mathrm{Cl}_{2}\right)$ then capped with a rubber septum. Separately, a solution of pentafluorophenylboronic acid $(8.0 \mathrm{mg}, 38 \mu \mathrm{mol}, 5$ equiv to $\left.[\mathrm{Pd}]_{\text {total }}\right)$ dissolved in THF- $d_{8}(0.2 \mathrm{~mL})$ was drawn into a $1 \mathrm{~mL}$ syringe; the needle tip was inserted into a rubber stopper to prevent air exposure during transport. Both the NMR tube and syringe were taken out of the box, and an initial NMR was acquired at $-70^{\circ} \mathrm{C}$. The boronic acid solution was then injected into the NMR tube while cooling in an acetone/dry ice bath $\left(-78^{\circ} \mathrm{C}\right)$. The NMR tube was shaken gently for $1 \mathrm{~min}$ after the addition then inserted in the NMR probe. ${ }^{1} \mathrm{H}, \mathrm{COSY}$, and NOESY/EXSY NMR spectra were acquired at $-70^{\circ} \mathrm{C}$. 


\section{Data availability}

Experimental procedures, characterization data, and DFT calculations are available in the Supplementary Information. Crystallographic data for 16 are deposited to the Cambridge Crystallographic Data Centre (CCDC) with the reference number 2105517. Additional data are available from the authors upon request.

\section{Acknowledgements}

We thank the NIH (R35GM128902) for financial support. We thank Phil Jeffrey for X-ray crystallographic data and Anthony Scavuzzo for assistance with DFT calculations.

\section{Author Contributions}

$S L, L C$, and $B C$ conceptualized the study and designed the experiments. SL and $L C$ conducted the experiments and prepared a draft of the manuscript. KD performed X-ray Absorption Spectroscopy (XAS) experiments and analysed the EXAFS data. IK and PL performed DFT calculations. BC finalized the manuscript. All authors contributed to several discussions related to this study and writing of this manuscript.

\section{Competing Interests}

The authors declare no competing interests.

\section{References}

1 Pd-catalysed cross-coupling reactions comprise $>70$ distinct transformations according to: Negishi, E.-i., Wang, G., Rao, H. \& Xu, Z. Alkyne Elementometalation-Pd-Catalyzed Cross-Coupling. Toward Synthesis of All Conceivable Types of Acyclic Alkenes in High Yields, Efficiently, Selectively, Economically, and Safely: "Green" Way. J. Org. Chem. 75, 3151-3182, (2010).

2 Tsuji, J. Palladium reagents and catalysts: new perspectives for the 21st century. (John Wiley \& Sons, 2006).

3 Echavarren, A. M.; Cárdenas, D. J., Mechanistic Aspects of Metal-Catalyzed C,C- and C,X-Bond-Forming Reactions. In Metal - Catalyzed Cross-Coupling Reactions, 2004; pp 1-40.

4 Trost, B. M. \& Crawley, M. L. Asymmetric Transition-Metal-Catalyzed Allylic Alkylations: Applications in Total Synthesis. Chem. Rev. 103, 2921-2944, (2003).

5 Barnard, C. F. J. Palladium-Catalyzed Carbonylation—A Reaction Come of Age. Organometallics 27, 54025422, (2008).

6 Chen, X., Engle, K. M., Wang, D. H. \& Yu, J. Q. Palladium (II) - catalyzed C-H activation/C-C cross coupling reactions: versatility and practicality. Angew. Chem. Int. Ed. 48, 5094-5115, (2009).

7 Lyons, T. W. \& Sanford, M. S. Palladium-catalyzed ligand-directed C- H functionalization reactions. Chem. Rev. 110, 1147-1169, (2010).

8 Tsuji, J. Synthetic Applications of the Palladium-Catalyzed Oxidation of Olefins to Ketones. Synthesis 1984, 369-384, (1984).

9 Takahashi, Y., Ito, T., Sakai, S. \& Ishii, Y. A novel palladium(0) complex; bis(dibenzylideneacetone)palladium(0). J. Chem. Soc. D: Chem. Commun., 1065-1066, (1970).

10 Sather, A. C. et al. A Fluorinated Ligand Enables Room-Temperature and Regioselective Pd-Catalyzed Fluorination of Aryl Triflates and Bromides. J. Am. Chem. Soc. 137, 13433-13438, (2015).

11 Littke, A. F., Dai, C. \& Fu, G. C. Versatile Catalysts for the Suzuki Cross-Coupling of Arylboronic Acids with Aryl and Vinyl Halides and Triflates under Mild Conditions. J. Am. Chem. Soc. 122, 4020-4028, (2000). 
12 Amatore, C. \& Pfluger, F. Mechanism of oxidative addition of palladium(0) with aromatic iodides in toluene, monitored at ultramicroelectrodes. Organometallics 9, 2276-2282, (1990).

13 Hartwig, J. F. \& Paul, F. Oxidative Addition of Aryl Bromide after Dissociation of Phosphine from a TwoCoordinate Palladium(0) Complex, Bis(tri-o-tolylphosphine)Palladium(0). J. Am. Chem. Soc. 117, 53735374, (1995).

14 Christmann, U. \& Vilar, R. Monoligated palladium species as catalysts in cross-coupling reactions. Angew. Chem. Int. Ed. Engl. 44, 366-374, (2005).

15 Andreu, M. G., Zapf, A. \& Beller, M. Molecularly defined palladium() monophosphine complexes as catalysts for efficient cross-coupling of aryl chlorides and phenylboronic acid. Chem. Commun., 2475-2476, (2000).

16 Krause, J. et al. 1,6-Diene Complexes of Palladium(0) and Platinum(0): Highly Reactive Sources for the Naked Metals and [L-MO] Fragments. J. Am. Chem. Soc. 121, 9807-9823, (1999).

17 Lee, H. G., Milner, P. J. \& Buchwald, S. L. An Improved Catalyst System for the Pd-Catalyzed Fluorination of (Hetero)Aryl Triflates. Org. Lett. 15, 5602-5605, (2013).

18 Lee, H. G., Milner, P. J., Colvin, M. T., Andreas, L. \& Buchwald, S. L. Structure and reactivity of [(L·Pd)n·(1,5cyclooctadiene)] ( $n=1-2)$ complexes bearing biaryl phosphine ligands. Inorg. Chim. Acta 422, 188-192, (2014)

19 Palani, V., Hugelshofer, C. L., Kevlishvili, I., Liu, P. \& Sarpong, R. A Short Synthesis of Delavatine A Unveils New Insights into Site-Selective Cross-Coupling of 3,5-Dibromo-2-pyrone. J. Am. Chem. Soc. 141, 26522660, (2019).

20 Fitton, P. \& Rick, E. A. The addition of aryl halides to tetrakis(triphenylphosphine)palladium(0). J. Organomet. Chem. 28, 287-291, (1971).

21 Fauvarque, J.-F., Pflüger, F. \& Troupel, M. Kinetics of oxidative addition of zerovalent palladium to aromatic iodides. J. Organomet. Chem 208, 419-427, (1981).

22 Barrios-Landeros, F. \& Hartwig, J. F. Distinct Mechanisms for the Oxidative Addition of Chloro-, Bromo-, and lodoarenes to a Bisphosphine Palladium(0) Complex with Hindered Ligands. J. Am. Chem. Soc. 127, 6944-6945, (2005).

23 Jover, J., Fey, N., Purdie, M., Lloyd-Jones, G. C. \& Harvey, J. N. A computational study of phosphine ligand effects in Suzuki-Miyaura coupling. J. Mol. Catal. A 324, 39-47, (2010).

24 Vikse, K., Naka, T., McIndoe, J. S., Besora, M. \& Maseras, F. Oxidative Additions of Aryl Halides to Palladium Proceed through the Monoligated Complex. ChemCatChem 5, 3604-3609, (2013).

25 Zheng, Q. et al. Capture of Reactive Monophosphine-Ligated Palladium(0) Intermediates by Mass Spectrometry. J. Am. Chem. Soc. 137, 14035-14038, (2015).

26 Ahlquist, M., Fristrup, P., Tanner, D. \& Norrby, P.-O. Theoretical Evidence for Low-Ligated Palladium(0): [Pd-L] as the Active Species in Oxidative Addition Reactions. Organometallics 25, 2066-2073, (2006).

27 Ahlquist, M. \& Norrby, P.-O. Oxidative Addition of Aryl Chlorides to Monoligated Palladium(0): A DFTSCRF Study. Organometallics 26, 550-553, (2007).

28 Li, Z., Fu, Y., Guo, Q.-X. \& Liu, L. Theoretical Study on Monoligated Pd-Catalyzed Cross-Coupling Reactions of Aryl Chlorides and Bromides. Organometallics 27, 4043-4049, (2008).

29 Vidossich, P., Ujaque, G. \& Lledos, A. Palladium monophosphine Pd(PPh3): is it really accessible in solution? Chem. Commun. 50, 661-663, (2014).

30 Chen, L., Ren, P. \& Carrow, B. P. Tri(1-adamantyl)phosphine: Expanding the Boundary of Electron-Releasing Character Available to Organophosphorus Compounds. J. Am. Chem. Soc. 138, 6392-6395, (2016).

31 Carrow, B. P. \& Chen, L. Tri (1-adamantyl) phosphine: exceptional catalytic effects enabled by the synergy of chemical stability, donicity, and polarizability. Synlett 28, 280-288, (2017).

32 Chen, L., Sanchez, D. R., Zhang, B. \& Carrow, B. P. "Cationic" Suzuki-Miyaura Coupling with Acutely BaseSensitive Boronic Acids. J. Am. Chem. Soc. 139, 12418-12421, (2017). 
33 Chen, L., Francis, H. \& Carrow, B. P. An "On-Cycle" Precatalyst Enables Room-Temperature Polyfluoroarylation Using Sensitive Boronic Acids. ACS Catal. 8, 2989-2994, (2018).

34 Lau, S. H. et al. Aryl Amination Using Soluble Weak Base Enabled by a Water-Assisted Mechanism. J. Am. Chem. Soc. 142, 20030-20039, (2020).

35 Teo, B.-K. Chemical applications of extended x-ray absorption fine structure (EXAFS) spectroscopy. Acc. Chem. Res. 13, 412-419, (1980).

36 Corker, J. et al. Catalytic Cleavage of the C-H and C-C Bonds of Alkanes by Surface Organometallic Chemistry: An EXAFS and IR Characterization of a Zr-H Catalyst. Science 271, 966, (1996).

37 Cox, P. A., Leach, A. G., Campbell, A. D. \& Lloyd-Jones, G. C. Protodeboronation of Heteroaromatic, Vinyl, and Cyclopropyl Boronic Acids: pH-Rate Profiles, Autocatalysis, and Disproportionation. J. Am. Chem. Soc. 138, 9145-9157, (2016).

38 Cox, P. A. et al. Base-Catalyzed Aryl-B(OH)2 Protodeboronation Revisited: From Concerted Proton Transfer to Liberation of a Transient Aryl Anion. J. Am. Chem. Soc. 139, 13156-13165, (2017).

39 Thomas, A. A. \& Denmark, S. E. Pre-transmetalation intermediates in the Suzuki-Miyaura reaction revealed: The missing link. Science 352, 329, (2016).

40 Thomas, A. A., Wang, H., Zahrt, A. F. \& Denmark, S. E. Structural, Kinetic, and Computational Characterization of the Elusive Arylpalladium(II)boronate Complexes in the Suzuki-Miyaura Reaction. J. Am. Chem. Soc. 139, 3805-3821, (2017).

41 Trotter, J. The crystal and molecular structure of phenanthrene. Acta Cryst. 16, 605-608, (1963).

42 Johnson, S. A., Huff, C. W., Mustafa, F. \& Saliba, M. Unexpected Intermediates and Products in the C-F Bond Activation of Tetrafluorobenzenes with a Bis(triethylphosphine)Nickel Synthon: Direct Evidence of a Rapid and Reversible C-H Bond Activation by Ni(0). J. Am. Chem. Soc. 130, 17278-17280, (2008).

43 Marshall, W. J. \& Grushin, V. V. Palladium(II) and Palladium(0) Complexes of BINAP(O) (2(Diphenylphosphino)-2'-(diphenylphosphinyl)-1,1'-binaphthyl). Organometallics 22, 555-562, (2003).

44 Ittel, S. D. \& Ibers, J. A. in Adv. Organomet. Chem. Vol. 14 (eds F. G. A. Stone \& Robert West) 33-61 (Academic Press, 1976).

45 Yadav, M. R. et al. The Suzuki-Miyaura Coupling of Nitroarenes. Journal of the American Chemical Society 139, 9423-9426, (2017).

46 Yokoyama, A. et al. Chain-Growth Polymerization for the Synthesis of Polyfluorene via Suzuki-Miyaura Coupling Reaction from an Externally Added Initiator Unit. J. Am. Chem. Soc. 129, 7236-7237, (2007).

47 Yokozawa, T., Suzuki, R., Nojima, M., Ohta, Y. \& Yokoyama, A. Precision Synthesis of Poly(3-hexylthiophene) from Catalyst-Transfer Suzuki-Miyaura Coupling Polymerization. Macromol. Rapid Commun. 32, 801-806, (2011).

48 Leone, A. K., Mueller, E. A. \& McNeil, A. J. The History of Palladium-Catalyzed Cross-Couplings Should Inspire the Future of Catalyst-Transfer Polymerization. J. Am. Chem. Soc. 140, 15126-15139, (2018).

49 Schoenebeck, F. \& Houk, K. N. Ligand-Controlled Regioselectivity in Palladium-Catalyzed Cross Coupling Reactions. J. Am. Chem. Soc. 132, 2496-2497, (2010).

50 Lyngvi, E., Sanhueza, I. A. \& Schoenebeck, F. Dispersion Makes the Difference: Bisligated Transition States Found for the Oxidative Addition of $\mathrm{Pd}(\mathrm{PtBu} 3) 2$ to $\mathrm{Ar}-\mathrm{OSO}_{2} \mathrm{R}$ and Dispersion-Controlled Chemoselectivity in Reactions with $\mathrm{Pd}[\mathrm{P}(\mathrm{Pr})(\mathrm{tBu} 2)]_{2}$. Organometallics 34, 805-812, (2015). 